( 2022 , The Authors. Published by Elsevier Inc. and Fass Inc. on behalf of the American Dairy Science Association ${ }^{\circledR}$. This is an open access article under the CC BY license (http://creativecommons.org/licenses/by/4.0/).

\title{
Genomic prediction in Nordic Red dairy cattle considering breed origin of alleles
}

\section{Ana Guillenea,* (i) Guosheng Su, Mogens Sandø Lund, and Emre Karaman (1)}

Center for Quantitative Genetics and Genomics, Aarhus University, 8830 Tjele, Denmark

\begin{abstract}
This study investigated the reliability of genomic prediction (GP) using breed origin of alleles (BOA) approach in the Nordic Red (RDC) population, which has an admixed population structure. The RDC population consists of animals with varying degrees of genetic materials from the Danish Red (RDM), Swedish Red (SRB), Finnish Ayrshire (FAY), and Holstein (HOL) because bulls have been used across the breeds. The BOA approach was tested using 39,550 RDC animals in the reference population and 11,786 in the validation population. Deregressed proofs (DRP) of milk, fat and protein were used as response variable for GP. Direct genomic breeding values (DGV) for animals in the validation population were calculated with (BOA model) or without (joint model) considering breed origin of alleles. The joint model assumed homogeneous marker effects and a single set of marker effects were estimated, whereas BOA model assumed heterogeneous marker effects, and different sets of marker effects were estimated across the breeds. For the BOA approach, we tested scenarios assuming both correlated (BOA_cor) and uncorrelated (BOA_uncor) marker effects between the breeds. Additionally, we investigated GP using a standard Illumina 50K chip and including SNP selected from imputed whole-genome sequencing (50K+WGS). We also studied the effect of estimating (co)variances for genome regions of different sizes to exploit the information of the genome regions contributing to the (co) variance between the breeds. Region sizes were set as 1 SNP, a group of 30 or 100 adjacent SNP, or the whole genome. Reliability of DGV was measured as squared correlations between DGV and DRP divided by the reliability of DRP. Across the 3 traits, in general, RS30 and RS100 SNP yielded the highest reliabilities. Including WGS SNP improved reliabilities in almost all scenarios (0.297 on average for $50 \mathrm{~K}$ and 0.307 on average
\end{abstract}

Received August 18, 2021.

Accepted November 23, 2021.

*Corresponding author: ana.guillenea@qgg.au.dk for 50K+WGS). The BOA_uncor (0.233 on average) was inferior to the joint model (0.339 on average), but the reliabilities obtained using BOA_cor (0.334 on average) in most cases were not significantly different from those obtained using the joint model. The results indicate that both including additional whole-genome sequencing SNP and dividing the genome into fixed regions improve GP in the RDC. The BOA models have the potential to increase the reliability of GP, but the benefit is limited in populations with a high exchange of genetic material for a long time, as is the case for RDC.

Key words: breed-specific effects, admixed cattle population, genomic selection

\section{INTRODUCTION}

Genomic prediction (GP; Meuwissen et al., 2001) is widely used to estimate breeding values of selection candidates. Genomic prediction relies on the consistency of linkage disequilibrium (LD) between the QTL and SNP between the reference and target populations. It has been successful in situations where the reference and target populations are from the same breed (VanRaden and Sullivan, 2010; Lund et al., 2011). SNP effects may differ between breeds because the pattern of LD over the genome may vary across different populations or their LD phase may be reversed (de Roos et al., 2008; Thomasen et al., 2013). Even QTL effects may differ between breeds if there are interactions between, for example, QTL and genetic background (Calus et al., 2018). Differences in SNP effects challenge both acrossand multi- breed predictions, in which the results differ depending on the models and traits analyzed (Erbe et al., 2012; Zhou et al., 2014; Calus et al., 2018).

Several statistical models have been proposed for GP considering breed origin of alleles (BOA). Some of them are based on distinguishing between alternate heterozygotes in estimating the effects of markers (Esfandyari et al., 2015), use breed-specific allele frequencies (Sevillano et al., 2017) or train the model based on pure and crossbred animals allowing the marker effects to be uncorrelated or correlated among breeds (Kara- 
man et al., 2021). When breeds are closely related, a combined reference population may lead to an increase in accuracies (Lund et al., 2014), whereas using the BOA assuming uncorrelated marker effects would not lead to additional benefits. In fact, it is likely to yield lower prediction accuracies because the reference to estimate breed-specific SNP effects is smaller than using a joint reference, as it only uses information from the single breed. However, when breeds are distantly related or unrelated, it is more appropriate to use a BOA model. A model that assumes that the marker effects are different between breeds but correlated (Varona et al., 2010; Karaman et al., 2021) is expected to perform well in both situations. This approach allows differentiating between the marker effects according to their breed origin, maximizing the use of the information of each breed.

Increasing marker density to the whole-genome level to reach a better LD between markers and QTL has been proposed to improve multibreed predictions (de Roos et al., 2008). However, in practice, the use of a high-density SNP chip (HD) led to little increase in reliability compared with the 50K SNP chip for both Nordic Red dairy cattle (RDC) and Nordic Holstein populations ( $\mathrm{Su}$ et al., 2012a). One possible reason could be that including a large number of markers without considering their functionality can lead to noise, limiting the reliability of GP (Pérez-Enciso et al., 2015). Moreover, de Roos et al. (2008) have shown that LD is conserved over long distances within breeds and short distances across breeds. van den Berg et al. (2016a) studied the influence of the distance between markers and causative mutations both within and across breeds, and they found that the loss in reliability quickly increased with the distance between markers and causative mutations, especially across breeds, and was influenced by the minor allele frequency of both causative mutations and markers. Therefore, to improve GP in a multibreed context, selected variants must be located very close to the causative mutation to ensure sufficient LD between marker and causal mutation in all breeds (van den Berg et al., 2016a). The standard medium-density SNP chips do not include all the markers that are in high LD with QTL across breeds. In that sense, including selected variants detected from whole-genome sequencing (WGS) data may leverage the accuracy of GP in multibreed scenarios.

Adjacent markers over a genome region are likely to be inherited together and in LD with the same QTL, and thereby, they may capture together the genetic variance at QTL (Sørensen et al., 2012; Gebreyesus et al., 2017). Therefore, the markers can be grouped such that variance of SNP within a group is assumed to be the same, whereas variance among the groups is allowed to differ, with a region size that reflects the heterogeneity of the variances over the genome (Gebreyesus et al., 2017). In single breed analysis, for production traits, models that considered heterogeneity over genome regions by grouping adjacent markers have substantially improved prediction reliability (Brøndum et al., 2012; Gebreyesus et al., 2017), confirming that it is reasonable to assume that the genomic (co)variance is unevenly distributed in the genome for the traits analyzed. Multibreed evaluations can benefit from region-wise grouping where LD is consistent across breeds, as the correlation of marker effects in regions with a similar LD pattern between breeds may be higher than in the rest of the genome (Lehermeier et al., 2015).

The population structure of the RDC is an admixture of Danish Red (RDM), Swedish Red (SRB) and Finnish Ayrshire (FAY) dairy cattle breeds, which have strong ties due to the use of common bulls (Brøndum et al., 2011). Especially the SRB and FAY breeds share a large part of their genetic origin, whereas the RDM also has genetic materials from Danish Holstein (HOL) and American Brown Swiss cattle (Team Avlsværdivurdering, 2009). Brøndum et al. (2011) investigated the accuracy of alternative reference populations for the RDC. According to their study, increasing the size of the reference population by joining 3 Red breeds led to higher accuracies than the individual homogeneous references. Thus, currently, a joint genetic evaluation is performed for RDC that ignores structure and assumes uniform marker effects across breeds, even when this leads to lower reliabilities than genetic evaluation for a homogeneous single breed as Holstein for comparable data sizes ( $\mathrm{Su}$ et al., 2012a). Our hypotheses were (1) accounting for breed-specific marker effect would be more accurate than assuming homogeneous marker effects across the breeds in RDC; (2) considering correlations of SNP effects between the breeds would allow for sharing information between breeds, which would lead to higher reliability than the model ignoring the correlations; (3) estimating region-specific (co)variances along the genome would increase reliability of GP compared with estimating a single (co)variance over the whole genome; and (4) reliability would be further improved by including WGS selected variants into the 50K chip.

Our primary objective was to compare the reliabilities from GP assuming homogeneous marker effects across the breeds (joint model) and accounting for breed-specific marker effects (BOA model) with or without considering correlations between the breeds. In addition, we investigated the effect of including regionspecific (co)variances along the genome and adding SNP selected from WGS data in GP. 


\section{MATERIALS AND METHODS}

\section{Nordic Red Cattle Data}

Animals and Phenotypes. We used deregressed proofs (DRP) of the RDC population on the Nordic scale for milk, fat and protein as response variables to predict direct genomic breeding values (DGV). In total, 51,336 animals (5,104 bulls and 46,232 cows) presented DRP, genotypes and sire registered in the pedigree. Among these animals, 9,300 were registered as RDM, 25,081 as FAY, and 16,955 as SRB. Although there were 1,849 registered sires in the pedigree, only 838 of these were among the 5,104 bulls. The 1,849 sires were used to group the animals to form validation and reference populations (explained later). The data were provided by the Nordic Cattle Genetic Evaluation (NAV; https://www.nordicebv.info/).

Genotypic Data. The bulls in the data set were genotyped using the conventional Illumina BovineSNP50 BeadChip array (50K). For cows, 23,245 were genotyped with the BovineLD BeadChip (Illumina) of which 4,122 were registered as RDM, 12,127 were registered as FAY, and 6,996 were registered as SRB. In addition, 22,987 cows were genotyped with customized LD chip which included markers of BovineLD BeadChip and the 6,685 markers from imputed WGS data selected by Denmark-Finland-Sweden (DFS) and France (FRA) (Brøndum et al., 2015, McLaren et al., 2016). Among these, 4,182 cows were registered as RDM, 10,422 were registered as FAY and 8,383 were registered as SRB. Both DFS and FRA SNP were identified for milk production and functional traits. The selection of these variants is described in detail in Liu et al. (2020a).

Preliminary pedigree analysis of the RDC population showed that the RDM has ties with the HOL due to the inclusion of HOL bulls in the breeding program of the RDM. Therefore, we decided to include a sufficient number of HOL bulls $(3,000)$ in our analysis. The HOL bulls were also genotyped with the $50 \mathrm{~K}$ chip. All marker positions were mapped onto the ARSUCD1.2 cattle genome assembly (Rosen et al., 2020). Reference alleles were aligned in RDC and HOL to combine their genotypes based on a standard 50K SNP set.

All genotyped animals with different chips were imputed to 50K+WGS and their genome were phased using FImpute v3.0 software (Sargolzaei et al., 2014) with default parameters and using pedigree. This was done chromosome-wise, separately for each breed. Only fully imputed/phased SNP were kept. Thus, 47,647 SNP which were common to RDC and HOL remained for GP. Among these SNP, 5,177 were WGS selected SNP, of which 1,600 were DFS, and 3,577 were FRA SNP.
Identification of Purebred Animals. To identify "pure animals" of each breed, we performed an ancestry analysis. We used all genotyped RDC bulls to perform the ancestry analysis, regardless of the presence of DRP. In total, 8,356 bulls were included in this analysis (5,356 RDC and 3,000 HOL). The bulls were allocated to purebred and admixed groups based on the predictions of breed proportions performed in ADMIXTURE v1.30 software (Alexander et al., 2009). The number of ancestral populations $(k)$ in ADMIXTURE was set to equal the expected number of breeds (RDM, FAY, $\mathrm{SRB}$, and HOL; i.e., $k=4$ ). Two different values of breed proportions were used as thresholds to define an animal as purebred: 0.93750 for RDM, FAY, and SRB, and 0.99997 for HOL. A relatively low threshold for RDC was chosen to ensure a sufficient number of purebred bulls from the 3 breeds, for breed origin assignments.

Assignment of BOA. Breed origin of alleles was inferred using ChromoPainterV2 software (Lawson et al., 2012), separately for each chromosome. In total, 1,820 selected "purebred" bulls in the ADMIXTURE analysis were used as donor population, among these, 751 were HOL, 244 were RDM, 760 were FAY and 65 were SRB. The rest of the RDC animals were defined as admixed, and their BOA were estimated in ChromoPainterV2, using the haplotypes of donor animals. Briefly, ChromoPainterV2 requires as input the SNP data for a set of admixed "recipient" chromosomes, the SNP data of the "donor" chromosome to represent the sources of admixture in the recipient chromosomes, and the genetic map with the recombination distance between each pair of contiguous SNP. It uses Hidden Markov Model forming each recipient chromosome as a mosaic of the donor chromosomes, capturing which donors explain the recipient DNA. The ChromoPainterV2 analyses were carried out maximizing over copying proportions using 10 expectation-maximization iterations with 3 samples per haplotype (Lawson et al., 2012). Genetic distances in morgans assumed that $1 \mathrm{Mb}$ corresponded to $1 \mathrm{cM}$, based on the recombination rate reported by Ma et al. (2015). The expected probability of each SNP coming from each of the pure breeds was used to infer the BOA by assigning each allele to certain pure breed for which the expected probability is the highest. We used the estimate of the breed origin of each allele to infer breed proportions for the admixed animals at individual level, in the proceeding analysis.

Generation of Reference and Validation Populations. Reference and validation populations were generated as follows. When more than half of the offspring of a sire were born on or before July 1, 2015, its offspring were considered in the reference popula- 
tion; otherwise, they were considered in the validation population. By implementing this method each group of half-sibs was placed together in the same population, either reference or validation. The 838 sires with DRP were also assigned based on their half-sibs. Consequently, the reference population consisted of 5,104 bulls and 34,446 cows, and the validation population consisted of 11,786 cows. Among the cows in the validation population, only 171 were daughters of a sire in the reference population. The number of animals per registered breed are presented in Table 1.

\section{Statistical Models}

We predicted DGV for the RDC population using 2 main approaches, namely joint and BOA. The joint model treats RDC as a single breed, and thereby, estimates a joint set of SNP effects across the original breeds. The BOA estimates breed-specific SNP effects assuming correlated or uncorrelated effects between the original breeds. To investigate the effects of including selected WGS SNP in GP, analyses were performed in 2 scenarios of SNP sets: $50 \mathrm{~K}: 42,470 \mathrm{SNP}$ and $50 \mathrm{~K}+\mathrm{WGS}$ : 47,647 SNP.

The joint model used to estimate SNP effects is as follows:

$$
\mathbf{y}=\mathbf{1} \mu+\mathbf{X b}+\mathbf{Z u}+\mathbf{e},
$$

where $\mathbf{y}$ is the vector of DRP of milk, fat, or protein for the reference animals, 1 is a vector of $1 \mathrm{~s}, \mu$ is the overall mean, $\mathbf{X}$ is the (column-centered) matrix of breed proportions based on an average breed proportion from the genotypes of an animal across all SNP, obtained from ChromoPainterV2. Breed proportions of donor animals are set as 1 in their respective breeds, $\mathbf{b}$ is the vector of fixed breed effects, $\mathbf{Z}$ is the matrix of centered genotypes based on current allele frequency in the reference animals, $\mathbf{u}$ is the vector of SNP effects, and $\mathbf{e}$ is the vector of random residuals.

We considered different region sizes as a fixed number of SNP to allow heterogeneous variances of SNP effects among different genome regions: 1 SNP (RS_1), 30 SNP (RS_30), 100 SNP (RS_100) and whole genome (RS_WG). To this end, $\mathbf{Z}$ and $\mathbf{u}$ were divided into $s$ nonoverlapping subsets of SNP at $k_{s}$ loci $(s=1$, $\ldots, \mathrm{S})$, and the priors were assigned to each subvector of SNP effects, $\mathbf{u}: \mathbf{u}_{\mathrm{s}} \mid \sigma_{s}^{2} \sim N\left(0, \mathbf{I} \sigma_{s}^{2}\right)$. The $\sigma_{s}^{2}$ were assigned to a scaled inverse chi-squared prior with degrees of freedom $\left(\mathrm{df}_{\mathrm{s}}\right)$ and a scale parameter $(\mathrm{S})$ : $\sigma_{s}^{2} \mid \mathrm{df}, \mathrm{S} \sim \chi^{-2}(\mathrm{df}, \mathrm{S})$. The parameters of this prior distribution were set as $\mathrm{df}=4$ and $\mathrm{S}=\frac{\sigma_{u}^{2}(\mathrm{df}-2)}{\mathrm{df}}(\mathrm{Ha}-$
Table 1. Number of Nordic Red animals in the reference and validation populations per registered population

\begin{tabular}{lrrrr}
\hline & \multicolumn{2}{c}{ Reference } & & Validation \\
\cline { 2 - 3 } $\begin{array}{l}\text { Registered } \\
\text { breed }^{1}\end{array}$ & Cows & Bulls & & Cows \\
\hline RDM & 6,058 & 996 & & 2,246 \\
FAY & 17,044 & 2,532 & & 5,505 \\
SRB & 11,344 & 1,576 & & 4,035 \\
Total & 34,446 & 5,104 & & 11,786 \\
\hline
\end{tabular}

${ }^{1} \mathrm{RDM}=$ Danish Red, FAY $=$ Finnish Ayrshire, $\mathrm{SRB}=$ Swedish Red, $\mathrm{HOL}=$ Holstein .

bier et al., 2011), where $\sigma_{u}^{2}$ is the SNP variance. The value of $\sigma_{u}^{2}$ was obtained from a preliminary analysis using only bulls' data, with the RS_WG. Given that BayesA assume that each SNP effect follows a normal distribution with null mean and a locus-specific variance, the RS_1 can be regarded as BayesA using the joint model. Contrary, the RS_WG can be regarded as SNP-BLUP because it assumes that all SNP have a null mean and a common variance.

The model to estimate breed-specific SNP effects using BOA is as follows (Karaman et al., 2021):

$$
\begin{gathered}
\mathbf{y}=\mathbf{1} \mu+\mathbf{X b}+\mathbf{Z}_{\mathrm{D}} \mathbf{u}_{\mathrm{D}}+\mathbf{Z}_{\mathrm{F}} \mathbf{u}_{\mathrm{F}}+\mathbf{Z}_{\mathrm{S}} \mathbf{u}_{\mathrm{S}} \\
+\mathbf{Z}_{\mathrm{H}} \mathbf{u}_{\mathrm{H}}+\mathbf{e}
\end{gathered}
$$

where $\mathbf{y}, \mathbf{1}, \mu, \mathbf{S}, \mathbf{b}$, and $\mathbf{e}$ are as described in Equation $1 ; \mathbf{Z}_{\mathrm{D}}, \mathbf{Z}_{\mathrm{F}}, \mathbf{Z}_{\mathrm{S}}$, and $\mathbf{Z}_{\mathrm{H}}$ are the matrices of breed-specific content of SNP for RDM, FAY, SRB, and HOL, and $\mathbf{u}_{\mathrm{D}}, \mathbf{u}_{\mathrm{F}}, \mathbf{u}_{\mathrm{S}}$, and $\mathbf{u}_{\mathrm{H}}$ are vectors of SNP effects for RDM, FAY, SRB, and HOL, respectively. These $\mathbf{Z}$ matrices were formed by assigning each allele to a certain pure breed for which the expected probability is the highest. The entry at a locus in a $\mathbf{Z}$ matrix, for instance $\mathbf{Z}_{\mathrm{D}}$, was the number of $A$ alleles ( 0,1 , or 2$)$ originating from $\mathrm{RDM}$ for an animal. Consequently, if an animal had $a a$ genotype or had no allele originating from RDM, the corresponding entry in $\mathbf{Z}_{\mathrm{D}}$ was zero. The same applies to other $\mathbf{Z}$ matrices for each breed. All columns of $\mathbf{Z}$ matrices were centered by subtracting the mean of each column before analysis.

Similar to the joint model, for the BOA models, all the matrices of genotypes $\left(\mathbf{Z}_{\mathrm{D}}, \mathbf{Z}_{\mathrm{F}}, \mathbf{Z}_{\mathrm{S}}, \mathbf{Z}_{\mathrm{H}}\right)$ and vectors of SNP effects $\left(\mathbf{u}_{\mathrm{D}}, \mathbf{u}_{\mathrm{F}}, \mathbf{u}_{\mathrm{S}}, \mathbf{u}_{\mathrm{H}}\right)$ were partitioned into $\mathrm{s}$ subsets each with $k_{s}$ loci $(1,30,100$, or whole genome). Each subvector of SNP was assigned a prior of a normal distribution separately for each breed $(j=\mathrm{D}, \mathrm{F}, \mathrm{S}, \mathrm{H}$ : $u_{j, s} \mid \sigma_{j, s}^{2} \sim N\left(0, \mathbf{I} \sigma_{j, s}^{2}\right)$. Consequently, the breed-specific SNP effects were assumed uncorrelated across the breeds, which means that the genetic correlations among the breeds were assumed to be zero 
(BOA_uncor). The $\sigma_{j, s}^{2}(s)$ were further assigned a scaled inverse chi-squared prior:

$$
\sigma_{j, s}^{2} \mid \mathrm{df}_{j}, \mathrm{~S}_{j} \sim \chi^{-2}\left(\mathrm{df}_{j}, \mathrm{~S}_{j}\right),
$$

where $\mathrm{df}_{j}=4$ and $\mathrm{S}_{j}=\frac{\sigma_{j}^{2}\left(\mathrm{df}_{j}-2\right)}{\mathrm{df}_{j}}$ (Habier et al., 2011). Here, $\sigma_{j}^{2}$ is the SNP variance for breed $j$, obtained from a preliminary analysis using only bulls' data, with the RS_WG.

Priors were also assigned to estimate breed-specific marker effects but assuming correlations between the breeds (BOA_cor). In that case, a multivariate normal distribution was assigned for the subvectors of SNP effects:

$$
\left[\mathbf{u}_{\mathrm{D}, \mathrm{S}}^{\prime}, \mathbf{u}_{\mathrm{F}, \mathrm{S}}^{\prime}, \mathbf{u}_{\mathrm{S}, \mathrm{S}}^{\prime}, \mathbf{u}_{\mathrm{H}, \mathrm{S}}^{\prime}\right]^{\prime} \mid \mathbf{B}_{\mathrm{S}} \sim N\left(0, \mathbf{B}_{\mathrm{S}} \otimes \mathbf{I}\right),
$$

where $\mathbf{I}$ is an identity matrix of size equal to $k_{s}$ if $k_{s}>$ 1 or 1 when $k_{s}=1$, and $\mathbf{B}_{\mathrm{S}}$ is as follows (Karaman et al., 2021):

$$
\mathbf{B}_{\mathrm{S}}=\left[\begin{array}{cccc}
\sigma_{\mathrm{D}, \mathrm{s}}^{2} & \sigma_{\mathrm{DF}, \mathrm{s}} & \sigma_{\mathrm{DS}, \mathrm{s}} & \sigma_{\mathrm{DH}, \mathrm{s}} \\
\sigma_{\mathrm{FD}, \mathrm{s}} & \sigma_{\mathrm{F}, \mathrm{s}}^{2} & \sigma_{\mathrm{FS}, \mathrm{s}} & \sigma_{\mathrm{FH}, \mathrm{s}} \\
\sigma_{\mathrm{SD}, \mathrm{s}} & \sigma_{\mathrm{SF}, \mathrm{s}} & \sigma_{\mathrm{S}, \mathrm{s}}^{2} & \sigma_{\mathrm{SH}, \mathrm{s}} \\
\sigma_{\mathrm{HD}, \mathrm{s}} & \sigma_{\mathrm{HF}, \mathrm{s}} & \sigma_{\mathrm{HS}, \mathrm{s}} & \sigma_{\mathrm{H}, \mathrm{s}}^{2}
\end{array}\right] .
$$

The diagonals and off-diagonals of $\mathbf{B}_{\mathrm{S}}$ are the breedspecific SNP variances and covariances, respectively. The $\mathbf{B}_{\mathrm{S}}$ were assigned an inverted Wishart (IW) distribution with a shape $\mathrm{v}_{\mathrm{B}}$ and a scale $\mathbf{V}_{\mathrm{B}}$ parameter matrix:

$$
\mathbf{B}_{\mathrm{S}} \mid \mathrm{v}_{\mathrm{B}}, \mathbf{V}_{\mathrm{B}} \sim I W\left(\mathrm{v}_{\mathrm{B}}, \mathbf{V}_{\mathrm{B}}\right)
$$

The inverted Wishart distribution is the multivariate version of the inverted Gamma distribution, and used as the conjugate prior for the variance-covariance matrix of a multivariate normal distribution (Sorensen and Gianola, 2002). We set $\mathbf{V}_{\mathrm{B}}=\left(\mathrm{v}_{\mathrm{B}}-4-1\right) \mathbf{B}$, where $\mathrm{v}_{\mathrm{B}}=7$, and $\mathbf{B}$ is the matrix of SNP (co)variances, which were obtained from a preliminary analysis using only bulls' data, and the RS_WG.

Given the assumption on the distribution of SNP effects in BayesA and SNP-BLUP, the RS_1 and RS_WG can be regarded as extensions of BayesA and SNP-BLUP, respectively, for multiple breeds using the BOA model.
In both models, random residuals were assumed to follow a normal distribution, $\mathbf{e} \sim N\left(0, \mathbf{D} \sigma_{e}^{2}\right)$, where $\sigma_{e}^{2}$ is the residual variance, and $\mathbf{D}$ is a diagonal matrix with elements $d_{i i}=1 / \mathrm{w}, \mathrm{w}$ is the weighting factor for the $i$ th $\mathrm{DRP}$ and is calculated as $\mathrm{w}=\mathrm{r}_{\mathrm{DRP}}^{2} /\left(1-\mathrm{r}_{\mathrm{DRP}}^{2}\right)$. The reliabilities of DRP were calculated as $\mathrm{r}_{\mathrm{DRP}}^{2}=\frac{\mathrm{ERC}}{\mathrm{ERC}+\lambda}$, where ERC is the effective record contribution and $\lambda=\frac{1-\mathrm{h}^{2}}{\mathrm{~h}^{2}}$. This weighting was used to account for heterogeneous residual variance due to different reliabilities of DRP $\left(\mathrm{r}_{\mathrm{DRP}}^{2}\right)$. The $\sigma_{e}^{2}$ was assigned to a scaled inverse chi-squared prior with degrees of freedom $\left(\mathrm{df}_{\mathrm{e}}\right)$ and a scale parameter $\left(\mathrm{S}_{\mathrm{e}}\right)$ :

$$
\sigma_{e}^{2} \mid \mathrm{df}_{e}, \mathrm{~S}_{e} \sim \chi^{-2}\left(\mathrm{df}_{e}, \mathrm{~S}_{e}\right) .
$$

For residual variances, $\mathrm{df}_{e}=4$ and $\mathrm{S}_{e}=\frac{\sigma_{e}^{2}\left(\mathrm{df}_{e}-2\right)}{\mathrm{df}_{e}}$, where $\sigma_{e}^{2}$ is the residual variance obtained also from the preliminary analysis of bulls' data for each model. Fixed effects were assigned flat priors.

The Markov-chain Monte Carlo algorithm with Gibbs sampling was run for 50,000 cycles to infer model parameters. The first 20,000 cycles were taken as burn in period and thus discarded. Every 10th cycle of the remaining 30,000 was saved, giving 3,000 posterior samples. The mean value of each parameter over the posterior samples was used as its estimate. The posterior samples are illustrated in Supplemental Figure S1 (https://dataverse.harvard.edu/dataset.xhtml ?persistentId=doi:10.7910/DVN/PFP90W, Guillenea, 2021) for milk analyzed with BOA_cor, SNP set 50K and RS_WG. In addition to marker effect estimates, we obtained, among others, the posterior distribution of $\mathbf{B}_{\mathrm{s}}$ (breed-specific SNP variances and covariances), which were used to calculate the correlation of SNP effects between breeds. The analyses were carried out using in-house software tools written in Julia programming language (Bezanson et al., 2017).

Evaluation of GP. After estimating marker effects in the reference population, the DGV of animals in the validation population were calculated by multiplying the genotype matrices (breed-specific matrices in BOA) with estimated marker effects (breed-specific SNP effects in BOA), and adding a fixed breed contribution $\left(\mathbf{X}_{v a l} \hat{\mathbf{b}}\right)$. The prediction reliability of GP for the RDC data was calculated as the squared correlation between DGV and DRP divided by the reliability of DRP for the animal in the validation data set, 


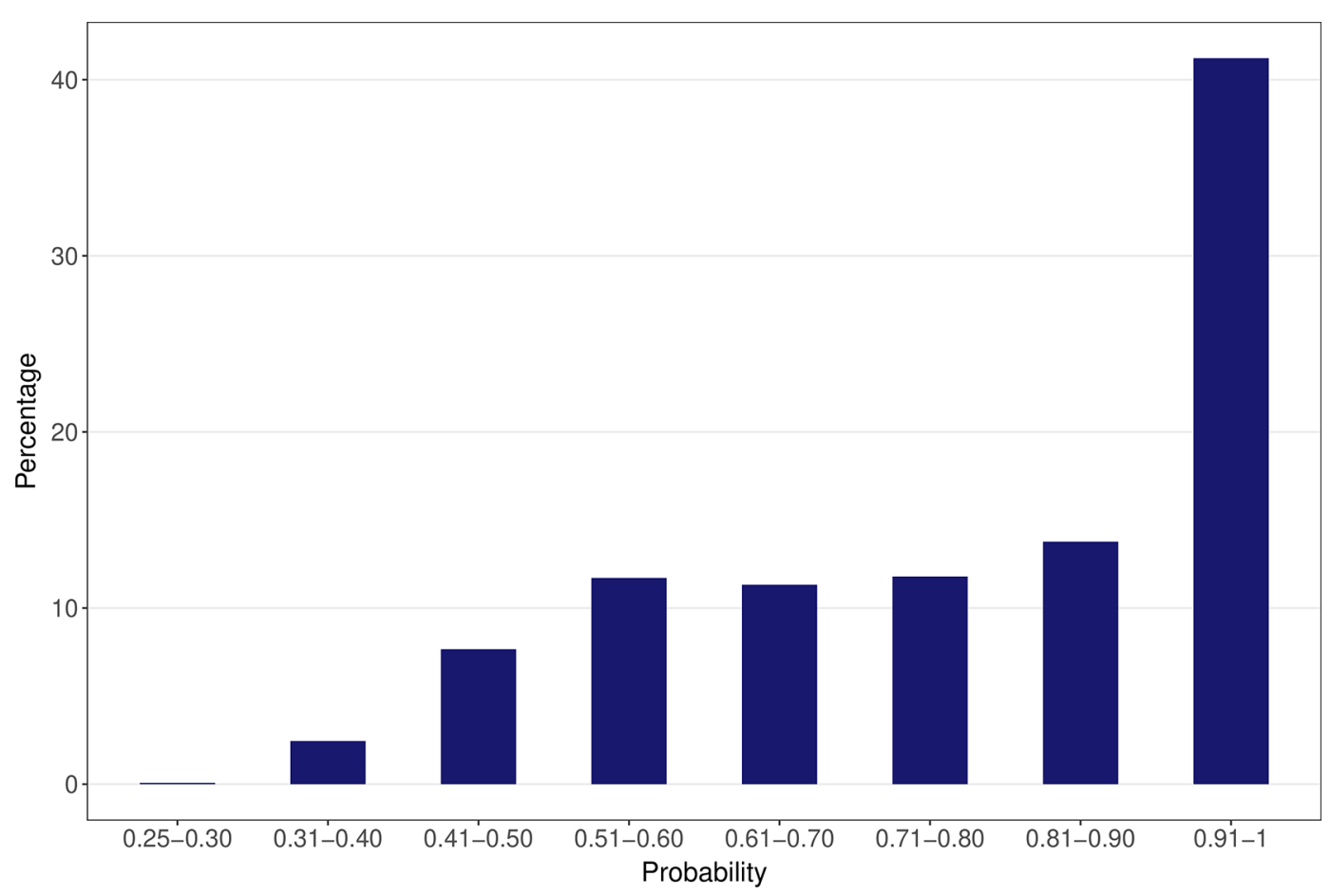

Figure 1. Distribution of probabilities to assign SNP to a particular breed using ChromoPainterV2 software (Lawson et al., 2012).

$\mathrm{r}_{\mathrm{DGV}}^{2}=\frac{\operatorname{cor}(\mathrm{DGV}, \mathrm{DRP})^{2}}{\mathrm{r}_{\mathrm{DRP}}^{2}}($ Su et al., 2012b). To facilitate statistical comparisons between the models, a bootstrapping procedure was applied. The bootstrapping involved computing reliabilities for 10,000 bootstrap samples of the validation animals of size equal to 11,786. The mean values of these 10,000 samples were reported in the paper as the reliabilities. We also calculated standard error of the mean with the bootstrap samples. Comparison between models, SNP sets, and region sizes for each trait were obtained from 2-tailed paired $t$-tests of the 10,000 samples. A Bonferroni adjustment of $P$ value was applied.

\section{RESULTS}

\section{Population Structure of the Nordic Red Cattle}

As shown in Table 2, the FAY breed had the lowest admixture level, with a contribution of other breeds of only $\sim 25 \%$. In contrast, the SRB breed has a contribution of $\sim 54 \% \mathrm{FAY}$ in its genome on average. The most admixed breed was the RDM, and it was the one that was genetically most associated with HOL (10\% of HOL, Table 2). However, the assignment of each allele in ChromoPainterV2 to a breed was not entirely accurate because only $41 \%$ of the alleles were assigned to a breed with high probability $(>0.90$; Figure 1$)$.

Table 2. Percentage of different populations across the genome of admixed Nordic Red Cattle individuals inferred from ChromoPainterV2

\begin{tabular}{|c|c|c|c|c|c|c|c|c|}
\hline \multirow{3}{*}{$\begin{array}{l}\text { Registered } \\
\text { breed }^{1}\end{array}$} & \multicolumn{8}{|c|}{ Percentage of each breed across genome } \\
\hline & \multicolumn{2}{|c|}{$\mathrm{RDM}$} & \multicolumn{2}{|c|}{ FAY } & \multicolumn{2}{|c|}{ SRB } & \multicolumn{2}{|c|}{ HOL } \\
\hline & Mean & SD & Mean & SD & Mean & SD & Mean & $\mathrm{SD}$ \\
\hline RDM & 0.34 & 0.15 & 0.32 & 0.15 & 0.23 & 0.09 & 0.10 & 0.05 \\
\hline FAY & 0.05 & 0.06 & 0.75 & 0.12 & 0.18 & 0.09 & 0.02 & 0.01 \\
\hline SRB & 0.13 & 0.02 & 0.54 & 0.13 & 0.30 & 0.10 & 0.03 & 0.02 \\
\hline
\end{tabular}

${ }^{1} \mathrm{RDM}=$ Danish Red, FAY $=$ Finnish Ayrshire, $\mathrm{SRB}=$ Swedish Red, HOL $=$ Holstein. 


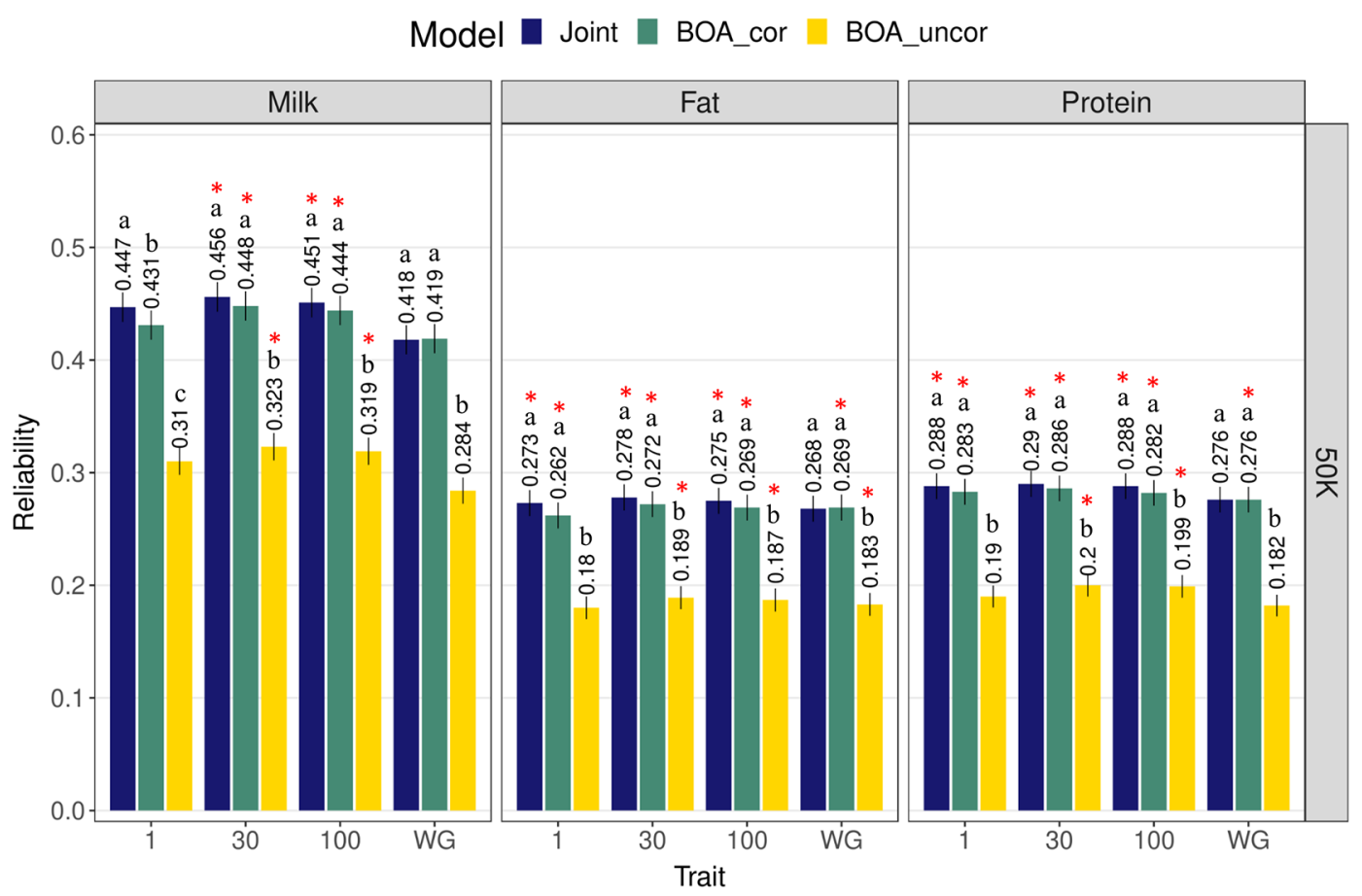

Figure 2. Average reliabilities and SE calculated with bootstrap sampling for the joint model and breed origin of alleles model assuming correlated (BOA_cor) or uncorrelated SNP effects between breeds (BOA_uncor) using SNP set 50K, for regions sizes of 1 SNP, 30 SNP, 100 SNP, and the whole genome (WG). Letters above the bars $(\mathrm{a}-\mathrm{c})$ indicate significant differences between models for each trait within the same region size. Asterisks indicate the region(s) size(s) with the highest reliability for each model within trait.

\section{Genomic Prediction for Nordic Red Cattle}

The reliabilities of GP using different models, traits and region sizes for SNP set 50K and 50K+WGS SNP are presented in Figures 2 and 3, respectively. Reliabilities of joint and BOA_cor were similar, and both models outperformed BOA_uncor for all scenarios. The joint model analyses were significantly superior to BOA_cor only for milk in the RS_1 (for SNP set 50K), and RS_1 and RS_30 (for SNP set 50K+WGS) scenarios.

In most cases, the reliabilities obtained with the BOA model, when the correlation between effects of the SNP of the different breeds were considered, were not significantly different from those obtained using the joint model. Averaged over the 3 traits, SNP sets and region sizes, reliability of GP from the joint model was 0.339 , which was $1.5 \%$ higher than the average from BOA_cor (0.334), and $45.5 \%$ higher than reliabilities from BOA_uncor (0.233).

The correlations of SNP effects among breeds inferred from BOA_cor were in general close to 1 , with the highest correlations between FAY and SRB and the lowest between the 3 Nordic breeds and HOL, as illustrated in Table 3 for milk, 50K SNP set in all the different region sizes (correlation averaged over all re- gions). The correlations tended to decrease to a certain extent when the region sizes were smaller.

Compared with using the $50 \mathrm{~K}$ chip, adding additional selected WGS SNP led to an improvement of $3.6 \%$ for milk and protein, and a smaller improvement $(2.4 \%)$ for fat. Furthermore, the increase was higher for reliabilities from BOA_uncor (4\% on average), than for BOA_cor and joint (3\% on average). Improvements in reliabilities for the inclusion of WGS SNP also differed among region sizes, with an increase of $2.6 \%$ for RS_1, $1.9 \%$ for RS_30, $2.8 \%$ for RS_100, and 6.0\% for RS_WG. In RS_WG, 50K+WGS SNP was always significantly superior to the 50K SNP. For the rest of the region sizes the differences depended on the trait and model (Supplemental Table S1; https://dataverse .harvard.edu/dataset.xhtml?persistentId=doi:10.7910/ DVN/PFP90W, Guillenea, 2021).

Averaged over the 3 traits, SNP sets and models, the reliability of RS_WG was 0.295 , RS_100 increased reliability by $3.8 \%$, RS_30 by $4.4 \%$ and RS_1 by $1.7 \%$. RS_30 and RS_100 were not statistically different, but in some scenarios these region sizes were significantly superior to RS_WG and RS_1. Pooled over the traits and SNP sets, average reliability of joint in RS_WG was 0.330 and was outperformed by $3.6,4.5$, and $3.2 \%$ by RS_100, RS_30 and RS_1, respectively. For BOA_cor 


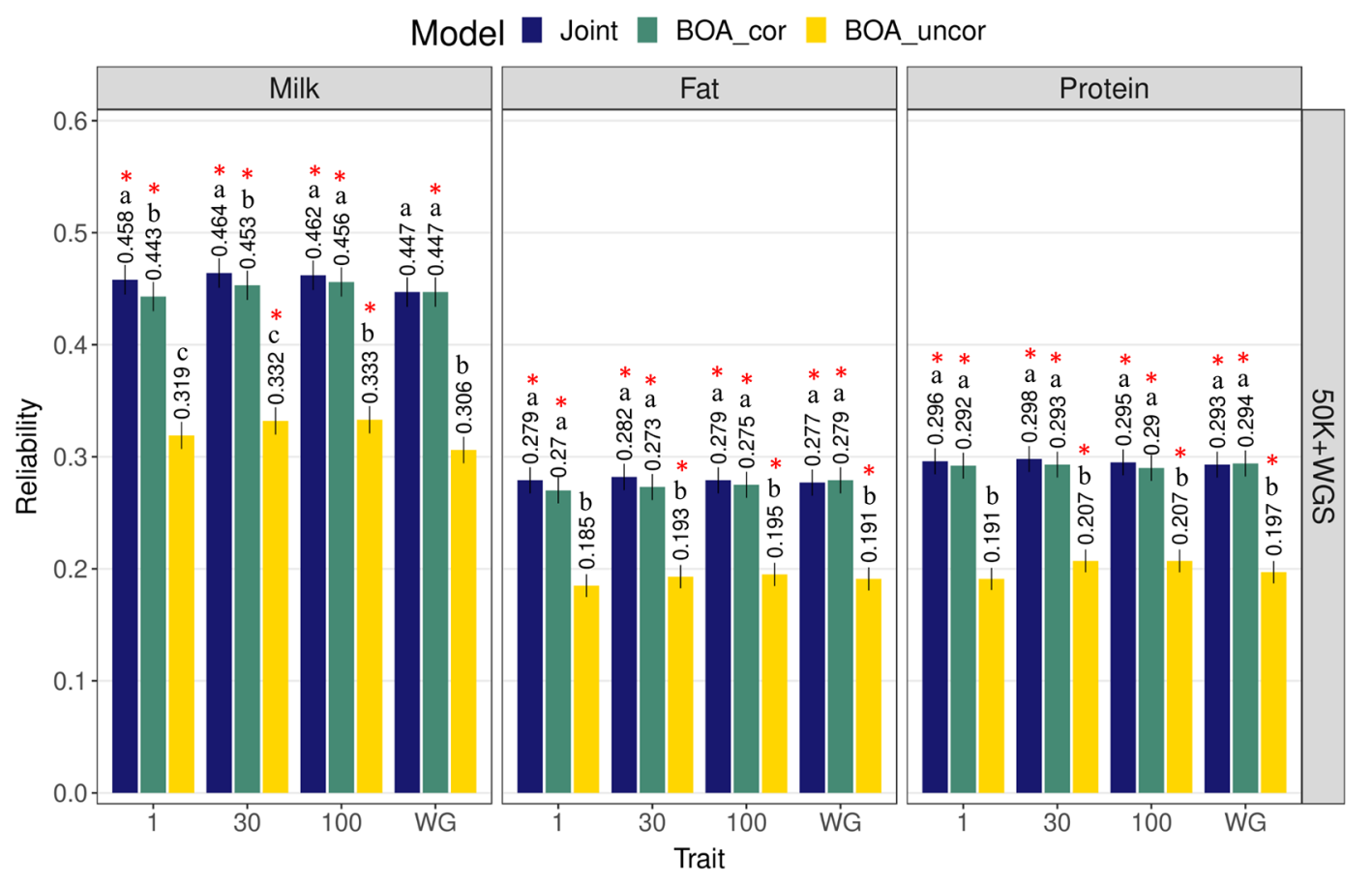

Figure 3. Average reliabilities and standard errors calculated with bootstrap sampling for the joint model and breed origin of alleles model assuming correlated (BOA_cor) or uncorrelated SNP effects between breeds (BOA_uncor) using SNP set 50K + whole-genome sequencing SNP (50K+WGS), for regions sizes of 1 SNP, 30 SNP, $100 \mathrm{SNP}$, and the whole genome (WG). Letters above the bars (a-c) indicate significant differences between models for each trait within the same region size. Asterisks indicate the region(s) size(s) with the highest reliability for each model within trait.

average reliability in RS_WG was 0.331 and there was a superiority of $1.1 \%$ for RS_100 and a superiority of $2.1 \%$ for RS_30, while there was a decrease of $0.3 \%$ for RS_1. For BOA_uncor, the gains were higher, especially in RS_100 and RS_30 with an average increase of 7.3 and $7.4 \%$, respectively, whereas the increase for RS_1 was of $2.4 \%$.

Table 3. Estimated correlation (averaged over all regions) between SNP effects of breeds in RDC population from BOA_cor model for milk, using SNP set 50K in different region sizes

\begin{tabular}{lllll}
\hline Region size $^{1}$ & Breed $^{2}$ & FAY & SRB & HOL \\
\hline RS_1 & RDM & 0.963 & 0.957 & 0.934 \\
& FAY & & 0.977 & 0.941 \\
RS_30 & SRB & & & 0.936 \\
& RDM & 0.977 & 0.972 & 0.959 \\
& FAY & & 0.985 & 0.962 \\
RS_100 & SRB & & & 0.958 \\
& RDM & 0.977 & 0.974 & 0.957 \\
RS_WG & FAY & & 0.985 & 0.960 \\
& SRB & & & 0.959 \\
& RDM & 0.992 & 0.988 & 0.987 \\
& FAY & & 0.995 & 0.989 \\
& SRB & & & 0.985 \\
\hline
\end{tabular}

${ }^{1}$ Region size $=$ number of SNP assigned the same variance $[1,30,100$ and WG (whole genome)].

${ }^{2} \mathrm{RDM}=$ Danish Red, FAY $=$ Finnish Ayrshire, $\mathrm{SRB}=$ Swedish Red, $\mathrm{HOL}=$ Holstein .
The advantage of splitting the genome differed across traits, and the increases in reliability over RS_WG for milk were $6.2,6.6$, and $3.8 \%$ for RS_100, RS_30 and RS_1, respectively, for protein the increases were 2.8 , 3.7 , and $1.4 \%$ and for fat, there was an increase of around 1\% for RS_100 and RS_30, but there was a decrease of the same magnitude for RS_1. However, in most cases, the differences between the regions were not statistically different (Figure 2 and Figure 3). The SE of the reliabilities ranged from 0.011 to 0.013 for joint and BOA_cor, and from 0.010 to 0.12 for BOA_uncor.

\section{DISCUSSION}

\section{Population Structure of the Nordic Red Cattle}

The highly admixed pattern found in the whole population made it difficult to define "pure" animals of the 3 Nordic breeds, where we used a threshold of $93.7 \%$ to keep a reasonable number of "pure" animals from each Nordic breed. Consequently, the assignments of each allele in ChromoPainterV2 was not entirely accurate, and only less than half of the alleles were assigned to their breed origin with a probability close to one. Previous analyses with ChromoPainterV2 have shown high accuracy in assigning alleles to their breed of origin in 
simulations with systematic crossing schemes (results not given).

ChromoPainterV2 analysis identified the presence of genomic traces of all the original breeds (RDM, FAY, SRB) and HOL in the RDC animals used in this study, and revealed a high degree of admixture in each of them, which has been reported in previous studies (Makgahlela et al., 2013; Gautason et al., 2019). The high proportion of FAY contributions in the genomes of SRB animals indicate a closer genetic relationship between these breeds, which is in concordance to the results reported by (Brøndum et al., 2011). The percentages of the different breeds in the genome of the admixed RDC confirmed the level of gene flow among the RDC breeds as well as from HOL to RDC, where the highest contribution of the HOL was to RDM, with an average of $10 \%$. Previous studies have reported that HOL was used in the breeding program of RDM, and consequently, they have a close genetic relationship (Makgahlela et al., 2013; Zhou et al., 2014; Gautason et al., 2019). The results of our ancestry analysis reflect the history of the Nordic breeds, which shows that especially the RDM and SRB are highly admixed. In particular, RDM has been characterized for an intense crossbreeding with American Brown Swiss, HOL, and the other Nordic Red dairy breeds in the late twentieth and early 21st century (Gautason et al., 2019), whereas SRB was formed in 1928 after joining Red pied Swedish with the Swedish Ayrshire (Bett et al., 2010). Afterward, genes of the other 3 Nordic breeds have continuosly been incorporated into SRB for the last 5 decades (Bett et al., 2010). This exchange of genetic material between RDM and SRB may explain the presence of HOL genes transmitted for RDM bulls into the SRB (Zhou et al., 2014), the same may apply for the presence of HOL in FAY. Inclusion of American Brown Swiss data could help to increase the accuracy of breed-origin assignments of alleles as well as GPs; however, we did not have genomic data on purebred animals of that breed.

\section{Genomic Prediction with and Without Considering Breed-specific SNP Effects}

Accounting for breed-specific SNP effects (BOA model) for the RDC population did not clearly improve the reliability of GP compared with a basic model (joint model) that assumes the same marker effects across breeds. The BOA_uncor uses less information than the other 2 approaches, because it uses only information from a single breed. The inclusion of data from admixed animals increases the size of the within-breed data to some extent, which, in some cases, could reduce the benefit of allowing correlation between breeds (Lehermeier et al., 2015). However, in our analysis, ignoring correlations (BOA_uncor) performed poorly compared with considering correlations (BOA_cor). The advantage of taking into account the correlations may be profound in the scenario of the high correlations among the breeds. The BOA_cor, as expected, had the ability to retrieve information from all breeds and performed similarly to the joint model. The inclusion of admixed animals in both BOA_cor and joint approaches, unlike BOA_uncor, can be considered as an increase in the size of the reference population. In that sense, many studies have reported higher accuracies when using a large reference population (Daetwyler et al., 2010; Cheng et al., 2018; Karaman et al., 2018). The correlations among the breeds estimated with BOA_cor were close to one even for the 3 Nordic breeds with HOL, which is higher than the correlations previously reported. Zhou et al. (2014) have reported genetic correlations between HOL and RDC in the range of 0.37 to 0.58 for production traits. It is possible that, in our study, uncertainty in the assignments of alleles to their breed of origin has led to overestimation of correlations between breeds.

Several studies showed that the benefit of considering breed-specific SNP effects decreased with increasing correlation of QTL and persistency of LD between the breeds, in other words, as the parental populations became more closely related (Ibanez-Escriche et al., 2009; Esfandyari et al., 2015).

In general, models considering breed-specific SNP effects tend to outperform models assuming the same marker effect across breeds in scenarios of large training population, small marker set and distantly related populations (Ibanez-Escriche et al., 2009; Esfandyari et al., 2015), whereas models only accounting for breed-specific allele frequencies in the construction of relationship matrices had shown limited effect on prediction (Makgahlela et al., 2014; Lourenco et al., 2016). In multibreed evaluations, SNP effects estimated separately within one breed and applied to other breeds performed poorly (Olson et al., 2012). A promising alternative method for GP in crossbred animals that has been proposed for the US dairy genetic evaluation is to estimate marker effects separately for each breed and use a weighted approach based on estimated breed proportions (VanRaden et al., 2020). This approach is convenient in programs where there are a few crossbreds generally not phenotyped. In populations with a large number of genotyped crossbred animals with phenotypic records, their data can also be used to estimate the effects of SNP, and BOA model facilitates this.

It should be noted that our BOA approach estimates breed-specific SNP effects, whereas it assumes that those effects are the same in a pure breed and its crosses with other breeds (admixed animals). Gene by genetic 
background interactions may alter the effects of the QTL (Lund et al., 2014), and consequently the effects of the SNP, resulting in a unique set of SNP effects for each breed. For the same reason, an allele originating from a pure breed may not have the same effect in an "admixed genome" and in the ancestral pure breed.

We did additional analyses where instead of assigning each allele to a certain original breed for which the expected probability is the highest, alleles were assigned to all possible breeds as a product of the allele count with the expected probabilities. Using the probabilities for potential breeds instead of assigning an allele to a single breed is a way to make breed-specific matrices more accurate. This approach resulted in higher reliabilities especially in the uncorrelated analysis (results not given). The correlated analysis uses information of all breeds, and account for correlation between them, thus it is not greatly affected by the wrong breed-origin assignments. Conversely, the uncorrelated depends more on the intrapopulation information. Therefore, it is more affected by errors in the assignments.

\section{Accounting for Heterogeneous (Co)variances Along the Genome}

In most cases, there were not significant differences for dividing the genome into regions, but in some scenarios RS_30 and RS_100 yielded significant improvements in reliabilities compared with the whole-genome analysis, as was reported in previous studies (Brøndum et al., 2012; Su et al., 2014; Gebreyesus et al., 2017). The joint and both BOA approaches showed similar patterns of increase in reliability in different region sizes. For the joint model, the idea behind grouping the SNP was that if some segments alone explain a larger/smaller part of the total variance compared with other regions, we expect an improvement of GP when considering this heterogeneity of the variance along the genome (Brøndum et al., 2012; Gebreyesus et al., 2017). The BOA approach can also benefit from regionwise correlations for segments where the LD phase is consistent across breeds.

Liu et al. (2020b) reported small differences between region sizes of 1 and $30 \mathrm{SNP}$ or region sizes of 100 SNP and the whole-genome analysis for production and fertility traits in Danish Jersey. Similarly, Karaman et al. (2021), in a simulation study, found negligible differences between 1 and 100 but the lowest accuracies were found for WG. These suggests that grouping the SNP has an advantage over the WG region size, but how to define an optimal region size needs to be further investigated. To efficiently use correlated information between the breeds using BOA models, local (region- wise) LD consistency between the breeds may be a factor to consider.

\section{Genomic Prediction Including Selected WGS SNP}

Compared with the 50K standard chip, the addition of the WGS SNP led to improvements in reliabilities. The inclusion of the WGS SNP led to higher gains in reliabilities than the reported by $\mathrm{Su}$ et al. (2012a) using a HD panel, even though a much smaller number of markers were added in our study. Su et al. (2012a) compared genomic prediction using either imputed HD markers or $54 \mathrm{~K}$ chip based on data from Nordic Holstein and RDC populations and they reported an increase in the reliability of DGV of $0.5 \%$ for protein when extending the SNP set to HD data, whereas results here showed an increase of $3 \%$ when simply combining the WGS markers with the standard 50K chip. Our results were consistent with the results of integrating QTL SNP in GP for Nordic Holstein and Nordic Red (Brøndum et al., 2015), WGS SNP in Danish Jersey (Liu et al., 2020a) and QTL SNP in a multibreed scenario (van den Berg et al., 2016b) for production traits. It is worth noting that the DFS WGS SNP were identified in a study that characterized the traits based on the economic weight of the trait in the Nordic index, which implies that more SNP for production traits were selected in comparison with other traits (Brøndum et al., 2015). Larger gains in reliabilities were obtained for milk, followed by protein and fat. Similarly, Liu et al. (2020b) reported that integrating SNP selected from sequencing data improved prediction for milk and protein, but resulted in limited or no improvement for fat, whereas previous studies reported larger gains for milk and fat, for which major QTL have been reported (Wang et al., 2012; Sahana et al., 2014). The 50K+WGS SNP yielded much higher reliabilities than the $50 \mathrm{~K}$ in the RS_WG $(\sim 6 \%)$ compared with the increase in other region size scenarios $(\sim 2 \%)$, but yet the reliability from RS_WG was lower than the rest of the region sizes in most of the scenarios in both the joint and BOA models (Figures 2 and 3). This indicates that the inclusion of selected markers became more important in WG region size models (i.e., SNP-BLUP model or the equivalent model GBLUP). One explanation is that, by the inclusion of the selected SNP, we increased the marker density around the QTL and the RS_WG benefit from that (Liu et al., 2020b), whereas the different region sizes already captured in a better way the variance of the QTL through the estimation of region-specific variances. Interestingly, for milk, for the 50K set, the RS_30 and RS_100 outperformed the other region sizes, whereas for the 50K+WGS SNP 
set, there were not significant differences among region sizes in most scenarios.

\section{Assignment of BOA}

In general, we did not observe the benefit of using BOA model over the joint model, which could possibly be due to BOA assignments being imprecise, and the accuracy of GP considering BOA rely on the accurate assignment of each SNP allele in the admixed animals to the ancestral breeds. The wrong assignment of the alleles makes it somewhat difficult to correctly estimate breed-specific SNP effects. For example, if the origin of alleles at a certain locus are switched (i.e., from A-B to B-A and vice versa), then the estimates of breedspecific alleles will be contaminated by information from the opposite breed. This contamination results in a higher similarity between estimated breed-specific effects, ultimately resulting in a higher correlation between breeds. We ran an additional simulation analysis (FileS3) with different percentages of alleles incorrectly assigned to a breed to assess the effect of the wrong assignments in the estimated correlation and accuracies of prediction. Details of the simulated populations are given in Supplemental Table S2 (https://dataverse .harvard.edu/dataset.xhtml?persistentId=doi:10.7910/ DVN/PFP90W, Guillenea, 2021). Our simulations showed that the estimated correlation between SNP effects tended to be higher as the percentage of errors in allele assignment increased (Supplemental Table S3, https://dataverse.harvard.edu/dataset.xhtml ?persistentId=doi:10.7910/DVN/PFP90W, Guillenea, 2021). The reliabilities of genomic breeding values from both BOA_cor and BOA_uncor were affected by wrong assignment of alleles regardless of the simulated correlation between breeds. BOA_uncor yielded lower reliabilities than the joint model when around $15 \%$ of the alleles were assigned to the wrong breed (Switched30; Supplemental Figure S2, https://dataverse.harvard .edu/dataset.xhtml? persistentId=doi:10.7910/DVN/ PFP90W, Guillenea, 2021).

Different approaches have been proposed to assign BOA, especially for simple crosses. Vandenplas et al. (2016) developed a procedure to trace breed origin to each allele in crossbreds using long-range phasing and it does not require prior knowledge of pedigree or close relationship between crossbred and purebred animals. This method was developed for a 2- or 3-way mating program and does not allow using crossbred sires, which limits its use in admixed populations such as $\mathrm{RDC}$, in which most of the bulls have different degree of contributions of the different Nordic breeds. In our ancestry analysis we identified 1,069 pure bulls of the Nordic breeds out of 5,356 included in the analysis.
There are also methods that use Hidden Markov Model, as ChromoPainterV2 (Lawson et al., 2012), which takes more time and in the case of the RDC, it showed difficulties in assigning alleles to their breeds of origin, though a relatively small number of purebred animals of some breeds were available at our breed proportion threshold. How to trace alleles back to their breed of origin efficiently and accurately is a challenging task for crossbreeding evaluation relying on BOA.

\section{CONCLUSIONS}

Our study compared reliability of GP of different statistical approaches (BOA and joint), and the effects of assuming region-specific (co)variances across the genome and of including WGS SNP in the $50 \mathrm{~K}$ set on reliability of GP in the RDC population. The results of this study indicate that even in populations with complicated admixture pattern, the BOA model can perform as well as the joint model. In addition, when the genetic correlation between breeds is high, assuming that marker effects are uncorrelated between breeds works poorly while considering the correlation between markers for different breeds, yields better results. In BOA models, the correct assignment of alleles to their breed origin is essential. Our results indicate that GP can be enhanced by accounting for region-specific variances across the genome and further improvements can be obtained by including WGS SNP in the standard $50 \mathrm{~K}$.

\section{ACKNOWLEDGMENTS}

This work has received funding from a PhD scholarship from the National Agency of Research and Innovation of Uruguay (INIA, Montevideo, Uruguay, POS_ EXT_2018_1_154296) and also from European Union's Horizon 2020 research and innovation programme-GenTORE-under grant agreement no. 727213. The authors are grateful to Aoxing Liu (Center for Quantitative Genetics and Genomics, Aarhus University, Tjele, Denmark) and Egill Gautason (Center for Quantitative Genetics and Genomics, Aarhus University, Tjele, Denmark) who helped with initial data preparation and with the new assembly map (ARSUCDI1.2) used in the analysis. The authors thank Gert Pedersen Aamand (SEGES, Aarhus, Denmark) and Ulrik Sander Nielsen (SEGES, Aarhus, Denmark) for assistance with data and valuable discussion. The authors also acknowledge Didier Boichard (INRAE, Animal Genetics and Integrative Biology, Jouy-en-Josas, France) for providing the FR SNP. Finally, the authors thank the editor and 2 anonymous referees for the valuable comments. The authors have not stated any conflicts of interest. 


\section{REFERENCES}

Alexander, D. H., J. Novembre, and K. Lange. 2009. Fast model-based estimation of ancestry in unrelated individuals. Genome Res. 19:1655-1664. https://doi.org/10.1101/gr.094052.109.

Bett, R., K. Johansson, E. Zonabend, B. Malmfors, J. Ojango, A. Okeyo, and J. Philipsson. 2010. Trajectories of evolution and extinction in the Swedish cattle breeds. in Proc. 9th World Congress Applied to Livestock Production, Leipzig, Germany. Gesellschaft für Tierzuchtwissenschaften e. V.

Bezanson, J., A. Edelman, S. Karpinski, and V. B. Shah. 2017. Julia: A fresh approach to numerical computing. SIAM Rev. 59:65-98. https://doi.org/10.1137/141000671.

Brøndum, R. F., E. Rius-Vilarrasa, I. Stranden, G. Su, B. Guldbrandtsen, W. F. Fikse, and M. S. Lund. 2011. Reliabilities of genomic prediction using combined reference data of the Nordic Red dairy cattle populations. J. Dairy Sci. 94:4700-4707. https://doi.org/10 .3168/jds.2010-3765.

Brøndum, R. F., G. Su, L. Janss, G. Sahana, B. Guldbrandtsen, D. Boichard, and M. S. Lund. 2015. Quantitative trait loci markers derived from whole genome sequence data increases the reliability of genomic prediction. J. Dairy Sci. 98:4107-4116. https://doi.org/ 10.3168/jds.2014-9005.

Brøndum, R. F., G. Su, M. S. Lund, P. J. Bowman, M. E. Goddard, and B. J. Hayes. 2012. Genome position specific priors for genomic prediction. BMC Genomics 13:543. https://doi.org/10.1186/1471 -2164-13-543.

Calus, M. P. L., M. E. Goddard, Y. C. J. Wientjes, P. J. Bowman, and B. J. Hayes. 2018. Multibreed genomic prediction using multitrait genomic residual maximum likelihood and multitask Bayesian variable selection. J. Dairy Sci. 101:4279-4294. https://doi.org/10 $.3168 /$ jds.2017-13366

Cheng, H., K. Kizilkaya, J. Zeng, D. Garrick, and R. Fernando. 2018. Genomic prediction from multiple-trait Bayesian regression methods using mixture priors. Genetics 209:89-103. https://doi.org/10 .1534 /genetics.118.300650.

Daetwyler, H. D., R. Pong-Wong, B. Villanueva, and J. A. Woolliams. 2010. The impact of genetic architecture on genome-wide evaluation methods. Genetics 185:1021-1031. https://doi.org/10.1534/ genetics.110.116855.

de Roos, A. P. W., B. J. Hayes, R. J. Spelman, and M. E. Goddard. 2008. Linkage disequilibrium and persistence of phase in HolsteinFriesian, Jersey and Angus cattle. Genetics 179:1503-1512. https: //doi.org/10.1534/genetics.107.084301.

Erbe, M., B. J. Hayes, L. K. Matukumalli, S. Goswami, P. J. Bowman, C. M. Reich, B. A. Mason, and M. E. Goddard. 2012. Improving accuracy of genomic predictions within and between dairy cattle breeds with imputed high-density single nucleotide polymorphism panels. J. Dairy Sci. 95:4114-4129. https://doi.org/10.3168/jds .2011-5019.

Esfandyari, H., A. C. Sorensen, and P. Bijma. 2015. A crossbred reference population can improve the response to genomic selection for crossbred performance. Genet. Sel. Evol. 47:76. https://doi.org/10 $.1186 / \mathrm{s} 12711-015-0155-\mathrm{z}$.

Gautason, E., A. A. Schönherz, G. Sahana, and B. Guldbrandtsen. 2019. Relationship of Icelandic cattle with Northern and Western European cattle breeds, admixture and population structure. Acta Agric. Scand. A Anim. Sci. 69:25-38.

Gebreyesus, G., M. S. Lund, B. Buitenhuis, H. Bovenhuis, N. A. Poulsen, and L. G. Janss. 2017. Modeling heterogeneous (co) variances from adjacent-SNP groups improves genomic prediction for milk protein composition traits. Genet. Sel. Evol. 49:89. https:// doi.org/10.1186/s12711-017-0364-8.

Guillenea, A. 2021. Supplemental Files: Genomic prediction in Nordic Red dairy cattle considering breed origin of alleles. JDS. Harvard Dataverse, V2. https://doi.org/10.7910/DVN/PFP90W.

Habier, D., R. L. Fernando, K. Kizilkaya, and D. J. Garrick. 2011. Extension of the Bayesian alphabet for genomic selection. BMC Bioinformatics 12:186. https://doi.org/10.1186/1471-2105-12-186.
Ibánez-Escriche, N., R. L. Fernando, A. Toosi, and J. C. M. Dekkers. 2009. Genomic selection of purebreds for crossbred performance. Genet. Sel. Evol. 41:12. https://doi.org/10.1186/1297-9686-41-12.

Karaman, E., M. S. Lund, M. T. Anche, L. Janss, and G. Su. 2018. Genomic prediction using multi-trait weighted GBLUP accounting for heterogeneous variances and covariances across the genome. G3 (Bethesda) 8:3549-3558.

Karaman, E., G. Su, I. Croue, and M. S. Lund. 2021. Genomic prediction using a reference population of multiple pure breeds and admixed individuals. Genet. Sel. Evol. 53:46. https://doi.org/10 $.1186 / \mathrm{s} 12711-021-00637-\mathrm{y}$.

Lawson, D. J., G. Hellenthal, S. Myers, and D. Falush. 2012. Inference of population structure using dense haplotype data. PLoS Genet. 8:e1002453. https://doi.org/10.1371/journal.pgen.1002453.

Lehermeier, C., C.-C. Schön, and G. de los Campos. 2015. Assessment of genetic heterogeneity in structured plant populations using multivariate whole-genome regression models. Genetics 201:323-337. https://doi.org/10.1534/genetics.115.177394.

Liu, A., M. S. Lund, D. Boichard, E. Karaman, S. Fritz, G. P. Aamand, U. S. Nielsen, Y. Wang, and G. Su. 2020a. Improvement of genomic prediction by integrating additional single nucleotide polymorphisms selected from imputed whole genome sequencing data. Heredity 124:37-49. https://doi.org/10.1038/s41437-019 -0246-7.

Liu, A., M. S. Lund, D. Boichard, E. Karaman, B. Guldbrandtsen S. Fritz, G. P. Aamand, U. S. Nielsen, G. Sahana, Y. Wang, and G. Su. 2020b. Weighted single-step genomic best linear unbiased prediction integrating variants selected from sequencing data by association and bioinformatics analyses. Genet. Sel. Evol. 52:48. https://doi.org/10.1186/s12711-020-00568-0.

Lourenco, D. A. L., S. Tsuruta, B. O. Fragomeni, C. Y. Chen, W. O. Herring, and I. Misztal. 2016. Crossbreed evaluations in single-step genomic best linear unbiased predictor using adjusted realized relationship matrices. J. Anim. Sci. 94:909-919. https://doi.org/10 $.2527 /$ jas.2015-9748.

Lund, M. S., A. P. de Roos, A. G. de Vries, T. Druet, V. Ducrocq, S. Fritz, F. Guillaume, B. Guldbrandtsen, Z. Liu, R. Reents, C. Schrooten, F. Seefried, and G. Su. 2011. A common reference population from four European Holstein populations increases reliability of genomic predictions. Genet. Sel. Evol. 43:43. https://doi.org/10 .1186/1297-9686-43-43.

Lund, M. S., G. Su, L. Janss, B. Guldbrandtsen, and R. F. Brøndum. 2014. Genomic evaluation of cattle in a multi-breed context. Livest. Sci. 166:101-110. https://doi.org/10.1016/j.livsci.2014.05.008.

Ma, L., J. R. O'Connell, P. M. VanRaden, B. Shen, A. Padhi, C. Sun, D. M. Bickhart, J. B. Cole, D. J. Null, G. E. Liu, Y. Da, and G. R. Wiggans. 2015. Cattle sex-specific recombination and genetic control from a large pedigree analysis. PLoS Genet. 11:e1005387. https://doi.org/10.1371/journal.pgen.1005387.

Makgahlela, M. L., I. Strandén, U. S. Nielsen, M. J. Sillanpää, and E. A. Mäntysaari. 2014. Using the unified relationship matrix adjusted by breed-wise allele frequencies in genomic evaluation of a multibreed population. J. Dairy Sci. 97:1117-1127. https://doi .org $/ 10.3168 /$ jds.2013-7167.

Makgahlela, M. L., E. A. Mäntysaari, I. Strandén, M. Koivula, U. Nielsen, M. Sillanpää, and J. Juga. 2013. Across breed multitrait random regression genomic predictions in the Nordic Red dairy cattle. J. Anim. Breed. Genet. 130:10-19. https://doi.org/10 .1111/j.1439-0388.2012.01017.x.

McLaren, W., L. Gil, S. E. Hunt, H. S. Riat, G. R. Ritchie, A. Thormann, P. Flicek, and F. Cunningham. 2016. The Ensembl Variant Effect Predictor. Genome Biol. 17:122. https://doi.org/10.1186/ s13059-016-0974-4.

Meuwissen, T. H., B. J. Hayes, and M. E. Goddard. 2001. Prediction of total genetic value using genome-wide dense marker maps. Genetics 157:1819-1829. https://doi.org/10.1093/genetics/157.4 .1819.

Olson, K. M., P. M. VanRaden, and M. E. Tooker. 2012. Multibreed genomic evaluations using purebred Holsteins, Jerseys, and Brown 
Swiss. J. Dairy Sci. 95:5378-5383. https://doi.org/10.3168/jds .2011-5006.

Pérez-Enciso, M., J. C. Rincón, and A. Legarra. 2015. Sequence-vs. chip-assisted genomic selection: Accurate biological information is advised. Genet. Sel. Evol. 47:43. https://doi.org/10.1186/s12711 $-015-0117-5$.

Rosen, B. D., D. M. Bickhart, R. D. Schnabel, S. Koren, C. G. Elsik, E. Tseng, T. N. Rowan, W. Y. Low, A. Zimin, and C. Couldrey. 2020. De novo assembly of the cattle reference genome with singlemolecule sequencing. Gigascience 9:giaa021.

Sahana, G., L. Janss, B. Guldbrandtsen, and M. Lund. 2014. Genetic architecture of milk, fat, protein, mastitis and fertility studied using NGS data in Holstein cattle. Proc. 10th World Congr. Genet. Appl. Livest. Prod. Vancouver, Canada. http://wcgalp .org/system/files/proceedings/2014/genetic-architecture-milk -fat-protein-mastitis-and-fertility-studied-using-ngs-data-holstein -cattle.pdf.

Sargolzaei, M., J. P. Chesnais, and F. S. Schenkel. 2014. A new approach for efficient genotype imputation using information from relatives. BMC Genomics 15:478. https://doi.org/10.1186/1471 -2164-15-478.

Sevillano, C. A., J. Vandenplas, J. W. M. Bastiaansen, R. Bergsma, and M. P. L. Calus. 2017. Genomic evaluation for a three-way crossbreeding system considering breed-of-origin of alleles. Genet. Sel. Evol. 49:75. https://doi.org/10.1186/s12711-017-0350-1.

Sorensen, D. and D. Gianola. 2002. Likelihood, Bayesian, and MCMC methods in quantitative genetics. Pages 55-59 in The Wishart and Inverse Wishart Distributions. Springer-Verlag.

Sørensen, L. P., L. Janss, P. Madsen, T. Mark, and M. S. Lund. 2012. Estimation of (co)variances for genomic regions of flexible sizes: Application to complex infectious udder diseases in dairy cattle. Genet. Sel. Evol. 44:18. https://doi.org/10.1186/1297-9686-44-18.

$\mathrm{Su}$, G., R. F. Brøndum, P. Ma, B. Guldbrandtsen, G. P. Aamand, and M. S. Lund. 2012a. Comparison of genomic predictions using medium-density $(\sim 54,000)$ and high-density $(\sim 777,000)$ single nucleotide polymorphism marker panels in Nordic Holstein and Red Dairy cattle populations. J. Dairy Sci. 95:4657-4665. https:// doi.org/10.3168/jds.2012-5379.

Su, G., O. F. Christensen, L. Janss, and M. S. Lund. 2014. Comparison of genomic predictions using genomic relationship matrices built with different weighting factors to account for locus-specific variances. J. Dairy Sci. 97:6547-6559. https://doi.org/10.3168/jds 2014-8210.

Su, G., P. Madsen, U. S. Nielsen, E. A. Mantysaari, G. P. Aamand, O. F. Christensen, and M. S. Lund. 2012b. Genomic prediction for Nordic Red Cattle using one-step and selection index blending. J. Dairy Sci. 95:909-917. https://doi.org/10.3168/jds.2011-4804.
Team Avlsværdivurdering. 2009. Årstatistik Avl - 2008/2009. Dansk Kvæg.

Thomasen, J. R., A. C. Sørensen, G. Su, P. Madsen, M. S. Lund, and B. Guldbrandtsen. 2013. The admixed population structure in Danish Jersey dairy cattle challenges accurate genomic prediction. J. Anim. Sci. 91:3105-3112. https://doi.org/10.2527/jas.2012 -5490 .

van den Berg, I., D. Boichard, B. Guldbrandtsen, and M. S. Lund. 2016a. Using sequence variants in linkage disequilibrium with causative mutations to improve across-breed prediction in dairy cattle: A simulation study. G3 (Bethesda) 6:2553-2561.

van den Berg, I., D. Boichard, and M. S. Lund. 2016b. Sequence variants selected from a multi-breed GWAS can improve the reliability of genomic predictions in dairy cattle. Genet. Sel. Evol. 48:83. https://doi.org/10.1186/s12711-016-0259-0.

Vandenplas, J., M. P. L. Calus, C. A. Sevillano, J. J. Windig, and J. W. M. Bastiaansen. 2016. Assigning breed origin to alleles in crossbred animals. Genet. Sel. Evol. 48:61. https://doi.org/10.1186/ s12711-016-0240-y.

VanRaden, P. M., and P. G. Sullivan. 2010. International genomic evaluation methods for dairy cattle. Genet. Sel. Evol. 42:7. https: //doi.org/10.1186/1297-9686-42-7.

VanRaden, P. M., M. E. Tooker, T. C. S. Chud, H. D. Norman, J. H. Megonigal Jr., I. W. Haagen, and G. R. Wiggans. 2020. Genomic predictions for crossbred dairy cattle. J. Dairy Sci. 103:1620-1631. https://doi.org/10.3168/jds.2019-16634.

Varona, L., C. Moreno, N. Ibanez-Escriche, and J. Altarriba. 2010. Whole genome evaluation for related populations. Pages $1-6$ in Proc. 9th World Congress on Genetics Applied to Livestock Production.

Wang, X., C. Wurmser, H. Pausch, S. Jung, F. Reinhardt, J. Tetens, G. Thaller, and R. Fries. 2012. Identification and dissection of four major QTL affecting milk fat content in the German HolsteinFriesian population. PLoS One 7:e40711. https://doi.org/10.1371/ journal.pone.0040711.

Zhou, L., M. S. Lund, Y. Wang, and G. Su. 2014. Genomic predictions across Nordic Holstein and Nordic Red using the genomic best linear unbiased prediction model with different genomic relationship matrices. J. Anim. Breed. Genet. 131:249-257. https://doi.org/10 $.1111 / j b g .12089$.

\section{ORCIDS}

Ana Guillenea @ https://orcid.org/0000-0002-5008-1043

Emre Karaman ำ https://orcid.org/0000-0003-1010-683X 